\title{
EAl Endorsed Transactions

\section{Performance Analysis on Popularity Based, Content Based and Collaborative Filtering Utilizing Recommendation Framework}

\author{
Deepkiran Munjal ${ }^{1, *}$, Anju Gera ${ }^{2}$ and Pawan Kumar Singh ${ }^{2}$ \\ ${ }^{1}$ Master of Computer Application, G. L Bajaj Institute of Technology and Management, Greater Noida, India \\ ${ }^{2}$ Computer Science and Engineering, G. L Bajaj Institute of Technology and Management, Greater Noida, India
}

\begin{abstract}
In today's computerized world, it has become an irritating undertaking to locate the substance of one's loving in an interminable assortment of substance that are being devoured like art, education, media and so on. Then again there has been a developing development among the computerized substance suppliers who need to connect the same number of clients on their administration as feasible for the most extreme time. A tune proposal is significant in our public activity because of its highlights, for example, in building smart cities recommending a lot of melodies to clients dependent on their advantage, or the popularity of the tunes. In this paper we are proposing a song suggestion framework that can prescribe song to another client just as the other existing clients. We use Popularity Based, Content Based separating, and Collaborative Filtering, which is a blend of application of communication systems, to develop a framework that gives progressively exact proposals concerning melodies.
\end{abstract}

\begin{abstract}
Keywords: Art education and media Recommender System, Popularity Based, Content Based filtering, Collaborative Filtering, smart
\end{abstract} cities, application of communication systems.

Handling Editor: Akshat Agrawal (Amity University Gurgaon, India)

Received on 24 July 2020, accepted on 13 August 2020, published on 25 August 2020

Copyright (C) 2020 Deepkiran Munjal et al., licensed to EAI. This is an open access article distributed under the terms of the Creative Commons Attribution license, which permits unlimited use, distribution and reproduction in any medium so long as the original work is properly cited.

doi: 10.4108/eai.18-8-2020.166001

*Corresponding author. Email:deepa.munjal@gmail.com

\section{Introduction}

Concerning a huge informational collection over the web, where the quantity of administrations are given, than in order to improve the issue of data over-burden, it is expected to channel, organize and effectively convey pertinent data, which has made an idle issue to numerous Internet clients. So as to convey clients with customized substance and administrations Recommender frameworks take care of this issue via looking through huge volume of enthusiastically created data. This proposition investigates the various attributes and possibilities of various expectation strategies in suggestion frameworks so as to fill in as a extent for research and practice in the field of proposal frameworks. Give $\mathrm{C}$ a chance to be set all things considered and let $\mathrm{S}$ be set of all conceivable recommendable things. Give $\mathrm{u}$ a chance to be an utility capacity evaluating the value of component $\mathrm{s}$ to client $\mathrm{c}$, i.e., u: $\mathrm{C} \mathrm{X} \mathrm{S} \rightarrow \mathrm{R}$, where $\mathrm{R}$ is a completely requested set. For every client $c \in C$, we need to pick things $s \in S$ that expand $\mathrm{u}$. The primary assignment is to appraise an utility capacity (u) that by configuration predicts how a client will like a thing. In light of Past conduct, Associations to different clients, Item similarity, Context and so forth.

With the ascent of advanced substance appropriation, individuals presently approach music assortments on an exceptional scale. Business music libraries effectively outperform 15 million tunes, which monstrously surpasses 
the listening ability of a specific individual. With a large number of melodies to browse, individuals some of the time feel overpowered. Therefore, an effectual music recommender framework is basic in light of a legitimate concern for both music specialist co-ops and clients. Clients will have no more agony to settle on choices on what to tune in while music organizations can keep up their client gathering and draw in new clients by improving client's fulfilment.

The fundamental goal of this paper is to give customized proposal, to build the degree of fulfilment of the client and to assist the clients with searching and listen the tunes in compelling and responsive way.

\section{Related Work}

There is hazardous development in the measure of existing advanced data and the quantity of guests to the Internet have made a potential test of data over-burden which hampers opportune access to things of enthusiasm on the Internet. Data recovery frameworks, for example, Google, Devil Finder and AltaVista have in part tackled this issue however prioritization and personalization (where a framework maps accessible substance to client's inclinations and inclinations) of data were missing. This has expanded the interest for recommender frameworks like never before previously. Recommender frameworks are gainful to both specialist co-ops and clients. They lessen expenses of finding and choosing things in an Online Music Portal. Recommendation frameworks have additionally demonstrated to improve basic leadership procedure and quality.

In Online Music Portal, recommender frameworks improve incomes, for the way that they are successful methods for selling more items. In logical libraries, recommender frameworks bolster clients by enabling them to move past inventory look. In this way, the need to utilize proficient and exact proposal strategies inside a framework that will give significant and trustworthy suggestions to clients can't be over-accentuated.

Engineering of requirements is one of the most basic phase of a product improvement process and inadequately actualized prerequisites building is one of the significant explanations behind venture disappointment [10]. Center prerequisites building exercises are elicitation and definition, quality confirmation, exchange, and discharge arranging [17]. These exercises can be upheld by suggestion advances, for instance, the (cooperative) proposal of prerequisites to partners taking a shot at comparable necessities [11] and the gathering based suggestion of necessities prioritizations [12]. Model: Content-based Recommendation based on content Requirements. In the accompanying, we will represent the use of substance based separating [13] with regards to necessities designing. A recommender can bolster invested individual, for instance, by prescribing prerequisites that have been characterized in effectively finished programming ventures (necessities reuse) or have been characterized by different partners of a similar undertaking (excess and reliance recognition). Table 3 gives a diagram of necessities characterized in a product venture. Every necessity is described by a class, the quantity of assessed individual days to execute the prerequisite, and a printed depiction.

Persuasive innovations [18] plan to trigger changes in a client's dispositions and comportment based on the ideas of human PC collaboration. The effect of enticing innovations can be fundamentally expanded by moreover coordinating proposal advancements into the plan of persuasive systems. Such a methodology pushes enticing advances ahead from a one-size-fits all way to deal with a customized domain where client explicit conditions are taken into translation while creating convincing messages [19]. Instances of the use of proposal innovations in the viewpoint of enticing frameworks are the execution of physical action while playing PC games [7] and moving programming engineers to improve the nature of their product modules [8]persuasive systems Games. Games concentrating on the inspiration of physical exercises incorporate extra reward systems to urge players to perform genuine real exercises. Berkovsky et al. [7] show the effective utilization of collaborative filtering recommendation innovations [6] for assessing the individual trouble of playing.

\section{Proposed System}

Music Recommendation System utilizes Popularity Based, Collaborative Filtering Based and Content Based Recommender to discover relationships among clients

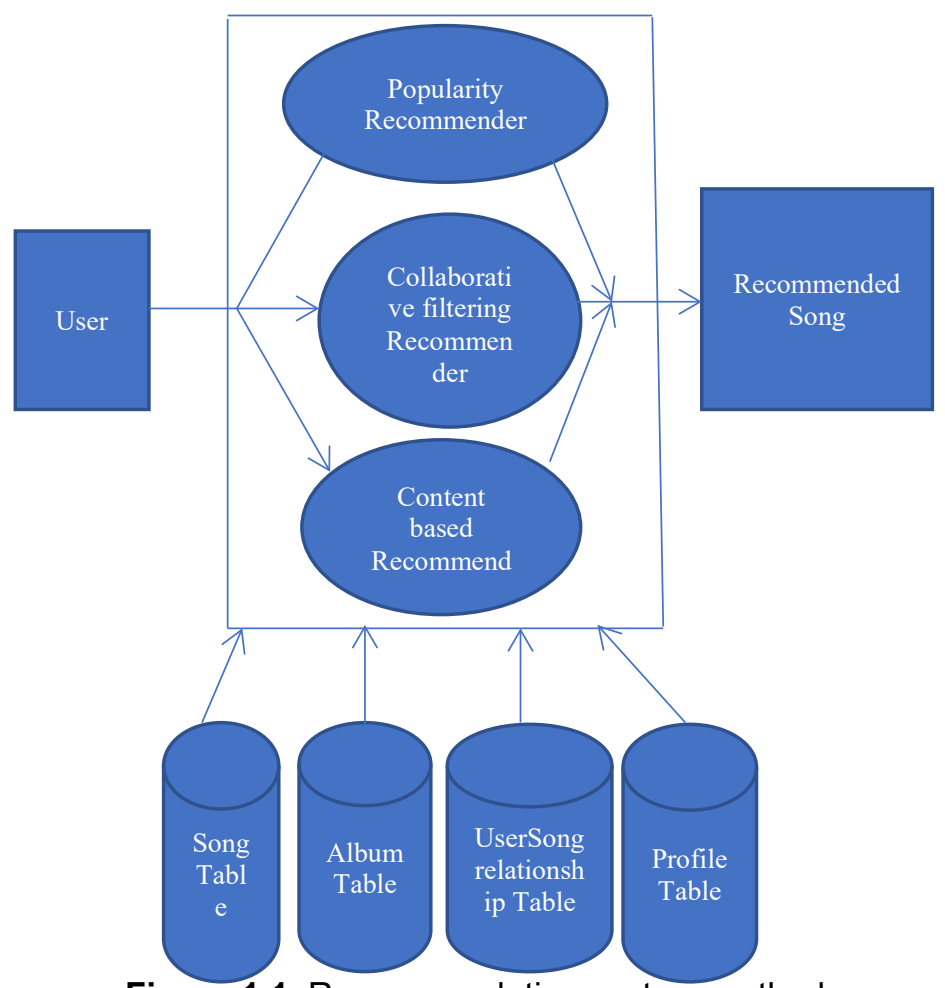

Figure 1.1. Recommendation system methods 
and tunes. Each of this recommender has certain preferences and impediments. This implementation is attempted to make a coordinated recommender which is blend of all these recommender to give

better suggestions. To defeat this, top spilling administrations utilize a mix of calculations to shape recommender framework.

Our first approach is to make a proposal system using content examination. First assignment is to remove the dataset suitable for desire. For this, we have taken the FMA and million tune dataset from columbia.edu and GitHub. Our Recommender System is a cross breed approach between Collaborative isolating, Popularity Based, and Content Based separating

The dataset was pre-processed using $\mathrm{R}$ and Python. The groups used for in $\mathrm{R}$ were readr, dplyr and caTools while pandas and numpy were used in python.

The Recommendation algorithms mainly follow collaborative filtering, content-based and filtering approaches:

1. Popularity Based : The most trifling suggestion calculation is to just present every melody in relative request of its prevalence bouncing those tunes previously devoured by the client, paying little mind to the client's taste profile.

2. Content Based Filtering: Use highlights of the two items just as clients so as to estimate whether a client will like an item or not.

3. Collaborative Filtering: It can be either client based or thing based. In client based underwriting, clients who tune comparable interests and will most likely tune in to similar tunes in future. In the thing based suggestion technique, melodies that are regularly tuned in by a similar client will in general be comparative and are bound to be listened reserved in future by some other client.

On basis of popularity based, Content based and Collaborative filtering the data is filtered and recommend the music for users. By combining the three evaluated result is filtered through and provide better recommendation.

\section{Result and Analysis}

The result outcome module is shown in this paper. It is implemented in python with the separate results of each filtering.

This evaluation is shown for popularity based recommender analysis.

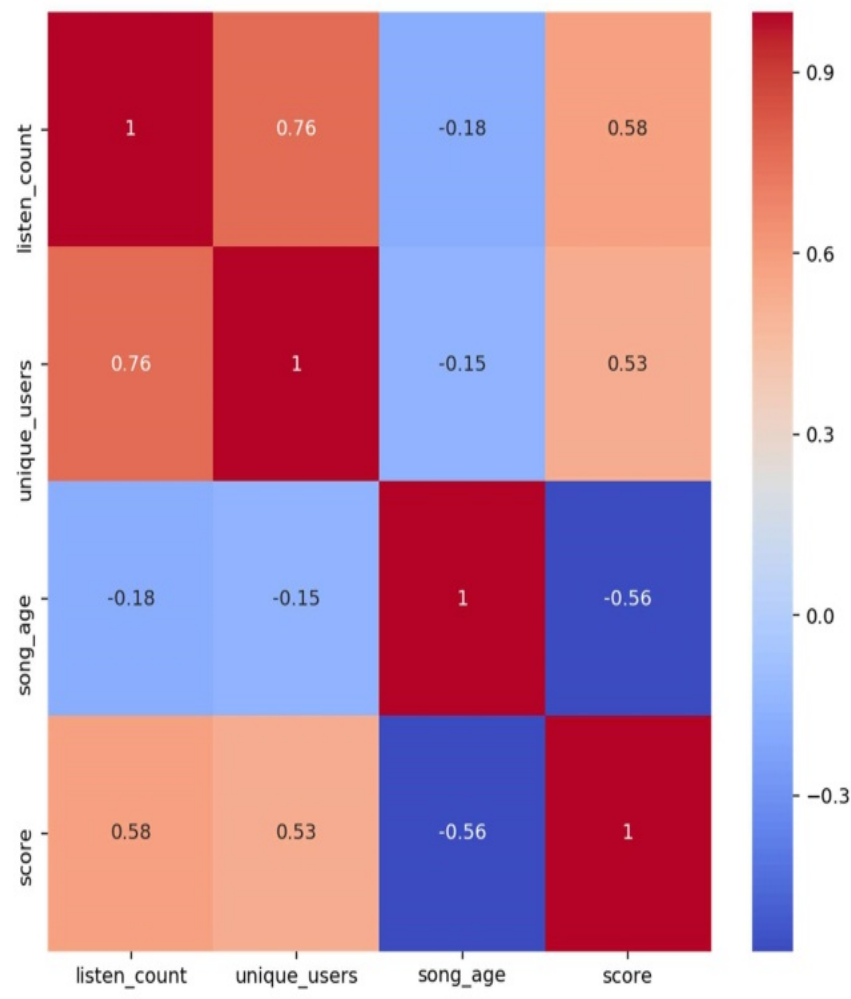

Figure 1.2. showing the correlation between features for song

To simply present each song in descending order of its popularity skipping those songs already consumed by the user, regardless of the user's taste profile.

\subsection{Popular Songs Scoring Formula}

Score $=([$ non_unique_user_listen_count* non_unique_users_weight] + [unique_user_listen_count $*$ unique_users_weight]) / song_age Whereunique_users_weight:1 non_unique_users_weight:0.25

Table1.1 Popular Songs score evaluation:

\begin{tabular}{|l|l|l|l|l|}
\hline $\begin{array}{l}\text { song_- } \\
\text { title }\end{array}$ & $\begin{array}{l}\text { listen_ } \\
\text { count }\end{array}$ & $\begin{array}{l}\text { unique_- } \\
\text { users }\end{array}$ & $\begin{array}{l}\text { song } \\
\text { age }\end{array}$ & score \\
\hline $\begin{array}{l}\text { Set Fire } \\
\text { To The }\end{array}$ & 5 & 3 & 2 & 1.75 \\
Rain & & & & \\
\hline
\end{tabular}




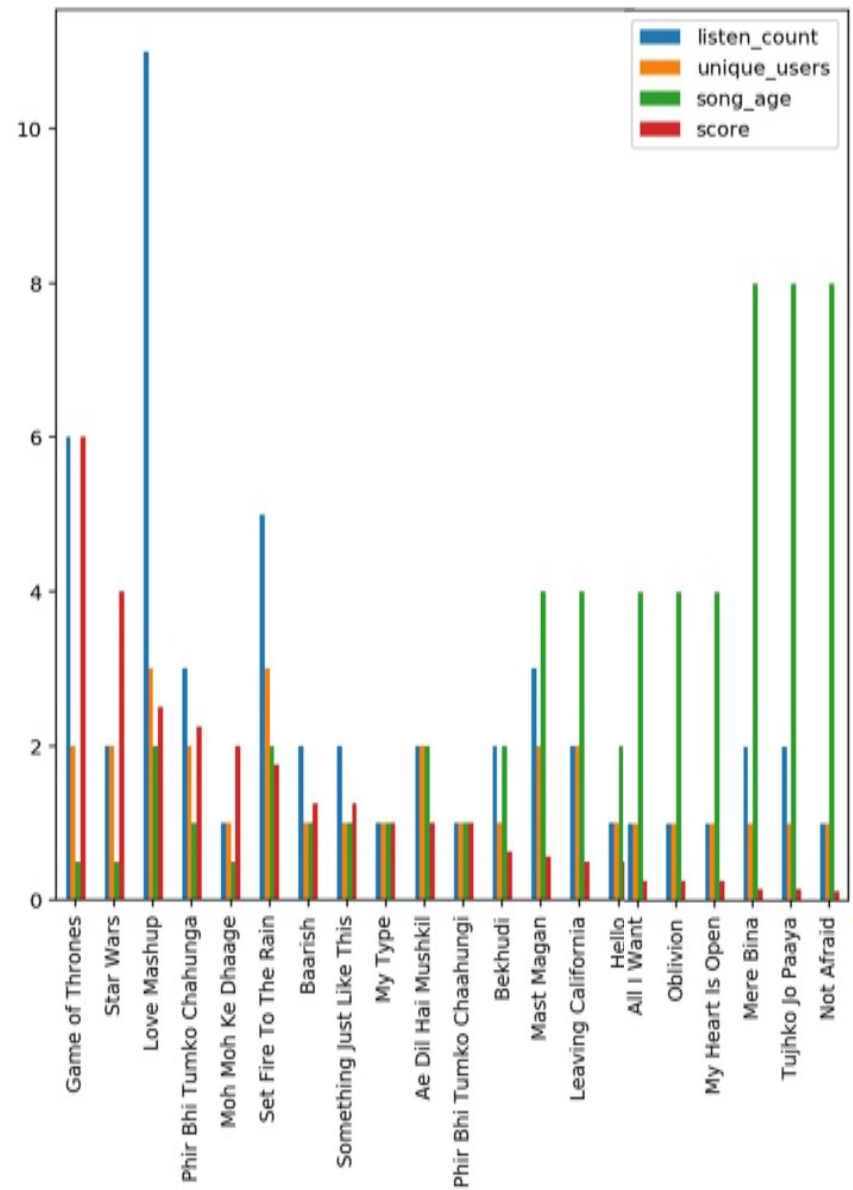

Figure 1.3. showing the values of various features of corresponding songs

It very well may be either client based or thing based. In client based suggestion, clients who tune in to similar melodies in the past will in general have comparative interests and will presumably tune in to similar tunes in future. In the thing based proposal procedure, tunes that are regularly tuned in by a similar client will in general be comparable and are bound to be listened together in future by some other client.

By controlling the informational collection, changing the learning set and testing set, changing a few parameters of the issue and breaking down the outcome, we acquire a great deal rehearsing aptitudes.

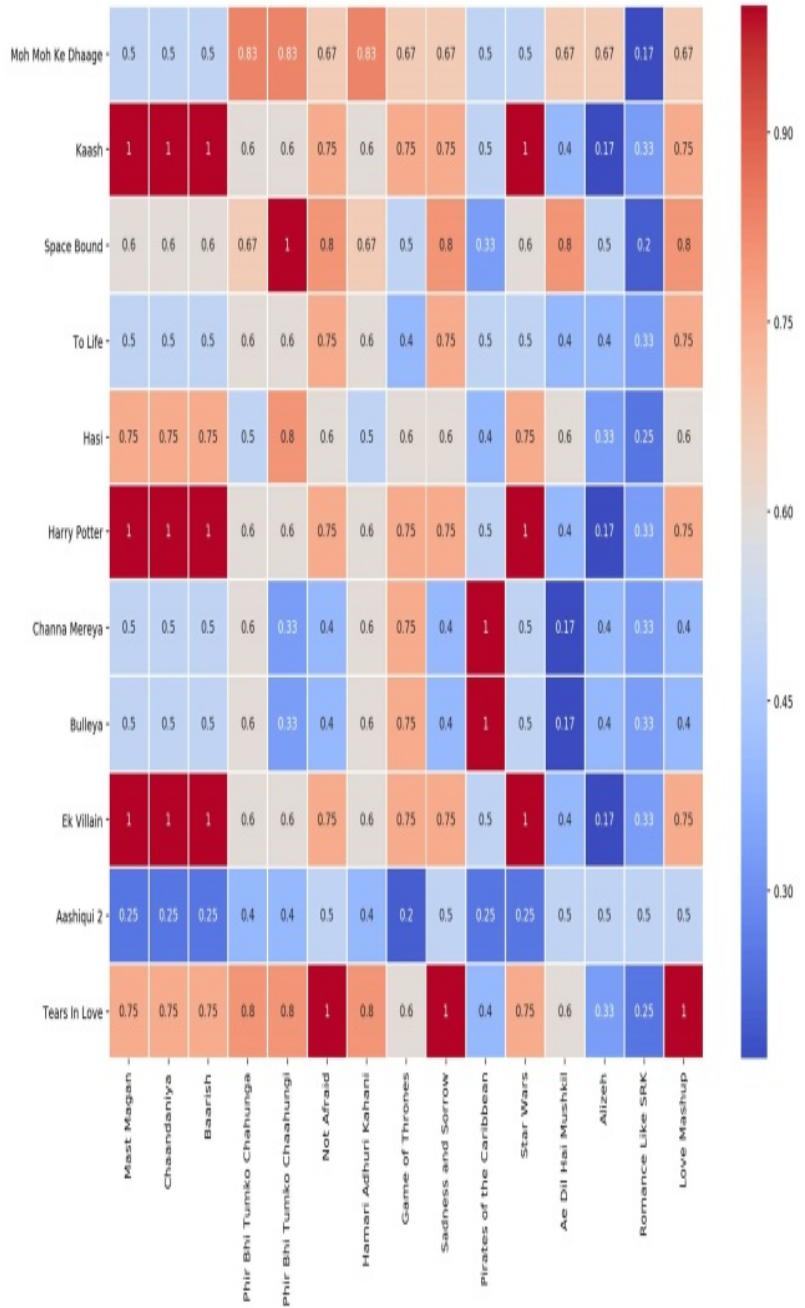

Figure 1.4. showing the Song Co-occurrence Matrix 


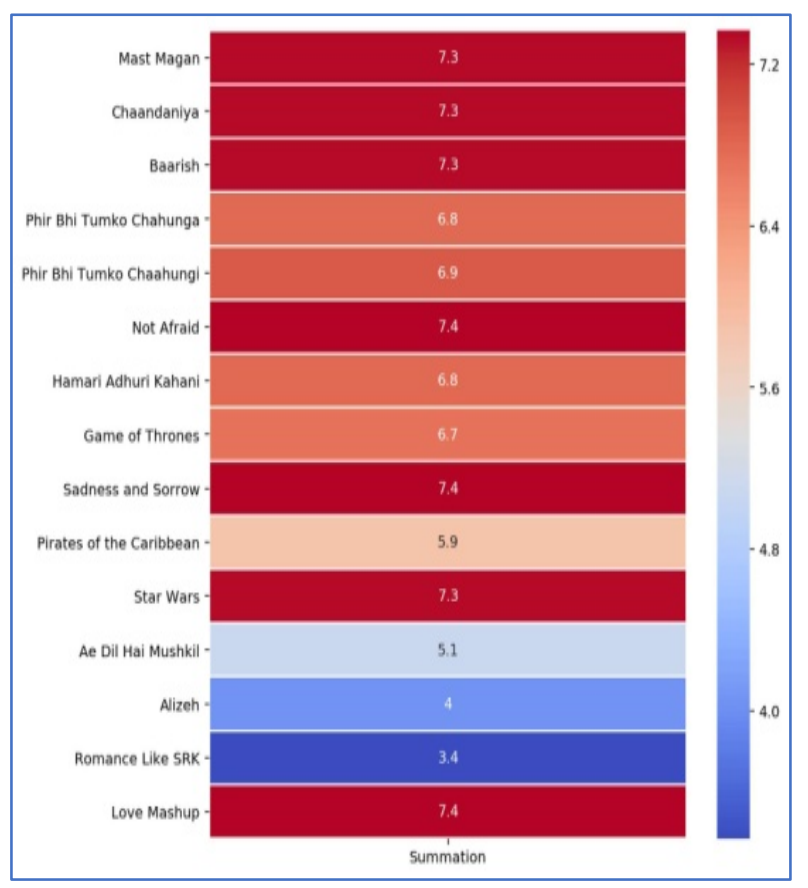

Figure 1.5. showing the Summation across columns in Song Co-occurrence Matrix

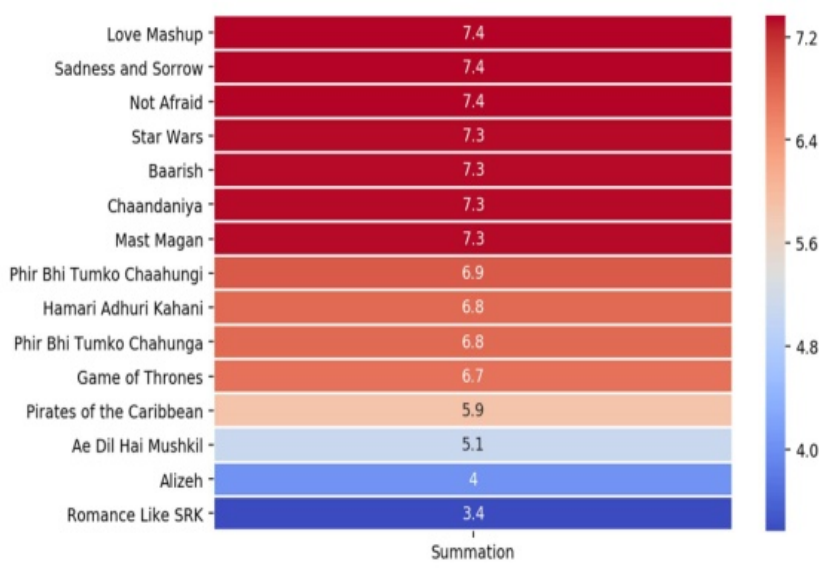

Figure 1.6. showing the Normalized Song Recommendations

\section{Conclusion}

In Music Recommender System there are various ways to deal with this issue and we become acquainted with certain calculations in detail and particularly the models that we have clarified previously. By utilized the informational index, changing the learning set and testing set, changing a few parameters of the issue and breaking down the outcome, we win a great deal rehearsing abilities. We have confronted a great deal of issues in managing tremendous dataset, how to investigate it in a superior way and we likewise experienced issues in some program configuration subtleties. In any case, with parcel of efforts, we have conquered these. As far as research, despite everything we have a ton to do to make our examinations a superior one.

Music Recommender System is such a wide, open and intricate matter that we can take a few activities and do significantly more tests in future. We likewise got the chance to understand that developing a recommender framework is certainly not a unimportant errand. The way that enormous scale dataset makes it troublesome in numerous perspectives. Right off the bat, suggesting pertinent tunes out of large dataset for various clients isn't a simple errand. Also, the metadata incorporates gigantic data and while investigating it, it is hard to dig important highlights for melody. Thirdly, in fact talking, preparing such a tremendous dataset is memory and CPU serious. Every one of these troubles because of the information and to the framework itself makes it all the more testing and furthermore increasingly appealing.

\section{Future Work}

In future, firstly we want to work upon segmentation of data set. There is a huge set of data available. To classify and segment data is the toughest job. We will work on this area to make data set clear and segregated. This will increase the performance of recommendation system more fast and accurate. For this we can apply many clustering algorithms.

In future the proposal framework can be utilized for different applications like in restorative field for directing the best medicinal analyse for fix of a patient and in future ( In medical field ) this will be relevant to work in space of man-made consciousness. Building capable suggestion set of rules and the comparing UIs requires a profound comprehension of human choice procedures. This objective can be accomplished by investigating existing mental plans of human basic leadership and their impact on the development of recommender frameworks.

\section{References}

[1] Book: Schafer, J.B., Konstan, J.A. \& Riedl, J. ECommerce Recommendation Applications. Data Mining and Knowledge Discovery 5, 115-153 (2001).

[2] Journal Article: Pazzani, M., Billsus, D, A Framework for Collaborative, Content-Based and Demographic Filtering Learning and revising user profiles, The identification of interesting web sites. Machine Learning, 1998.

[3] Conference: H. F. Hofmann and F. Lehner, "Requirements engineering as a success factor in software 
projects," in IEEE Software, vol. 18, no. 4, pp. 58-66, JulyAug. 2001.

[4] Book Chapter: Fogg, Persuasive Technology - Using Computers to Change What We Think and Do. Morgan Kaufmann Publishers, 2003.

[5] Journal Article: Schafer J.B., Konstan J.A., Riedl J.T. (2006) Recommender Systems for the Web. In: Geroimenko V., Chen C. (eds) Visualizing the Semantic Web. Springer, London..

[6] Conference: Berkovsky, S., Freyne, J., Coombe, M., Bhandari, D.: Recommender algorithms in activity motivating games. ACM Conference on Recommender Systems (RecSys'09), 2010.

[7] Journal Article: Mobasher, B., Cleland-Huang, J, Using data mining and recommender systems to scale up the requirements process, Recommender Systems in Requirements Engineering. AI Magazine 32(3), 2011.

[8] Journal Article: elfernig, A., Zehentner, C., Ninaus, G., Grabner, H., Maalej, W., Pagano, D., Weninger, L., Reinfrank, F.: Using Answer Set Programming for Feature Model Representation and Configuration, 2011.

[9] Journal Article: Janssen, J., Broek, E., Westerink, J.: Tune in to your emotions: a robust personalized affective music player. User Modeling and User-Adapted Interaction (UMUAI) .2011.

[10] Conference: Adomavicius, G., Bockstedt, J., Curley, S., Zhang, J.: Recommender systems, consumer preferences, and anchoring effects. In: RecSys 2011 Workshop on Human Decision Making in Recommender Systems, 2011.

[11] Conference: Pribik, I., Felfernig, A.: Towards Persuasive Technology for Software Development Environments: An Empirical Study. In: Persuasive Technology Conference, 2012.

[12] Journal Article: Berkovsky, S., Freyne, J., OinasKukkonen, H.: Influencing Individually: Fusing Personalization and Persuasion. ACM Transactions on Interactive Intelligent Systems 2(2), 2012

[13] Conference: Lalita Sharma and Anju Gera A Survey of Recommendation System: Research Challenges International Journal of Engineering Trends and Technology (IJETT) - Volume 4 May, 2013

[14] Conference: Shraddha B. Shinde, Mrs. M. A Potey,Research Paper Recommender System Evaluation Using Coverage, International Research Journal of Engineering and Technology (IRJET), 2016.

[15] Conference: hinde U., and Shedge R. Comparative Analysis of Collaborative Filtering Technique, IOSR Journal of Computer Engineering, Volume 10, 2013.

[16] Conference: Jiang, C., \& He, Y. (2016). Smart-DJ: Context-Aware Personalization for Music Recommendation on Smartphones. 2016 IEEE 22nd International Conference on Parallel and Distributed Systems (ICPADS)

[17] Journal Article: Haruna K, Akmar Ismail M, Damiasih D, Sutopo J, Herawan T, A collaborative approach for research paper recommender system. PLoS ONE 12(10): e0184516, 2017.

[18] Conference: Pirkka Åman, Lassi A. Liikkanen, Interacting with Context Factors in Music Recommendation and Discovery, Computer Science International Journal of Human-Computer Interaction, 2017.

[19] Conference: Åman, Pramila M. Chawan Int. Journal of Engineering Research and Application www.ijera.com ISSN : 2248-9622, Vol. 8, Issue5, May 2018 